\title{
Las mutaciones del gen CARD15 presentan escasa relación con los fenotipos de la enfermedad de Crohn en Asturias
}

\author{
L. Rodrigo, J. Martínez-Borra ${ }^{1}$, J. A. Garrote ${ }^{2,3}$, P. Niño, A. J. León ${ }^{3}$, S. Riestra, D. Bernardo 3 , M. Barreiro \\ y E. Arranz
}

Servicios de Aparato Digestivo e ${ }^{1}$ Inmunología. Hospital Universitario Central de Asturias. Oviedo. ${ }^{2}$ Unidad de Investigación. ${ }^{3}$ Hospital Clínico Universitario de Valladolid y Grupo de Estudio de Inmunología de las Mucosas. Departamentos de Pediatría e Inmunología. Universidad de Valladolid. ${ }^{4}$ Servicio de Aparato Digestivo. Hospital Clínico Universitario de Santiago de Compostela

\section{RESUMEN}

Introducción: la asociación entre las mutaciones del gen CARD15 y la susceptibilidad genética para la enfermedad de Crohn (EC) se ha confirmado en diversos estudios, con amplias variaciones observadas a nivel mundial, tanto geográficas como étnicas.

Objetivos: analizar la prevalencia de gen CARD 15 y sus polimorfismos en pacientes con EC en Asturias y su posible correlación con los diversos fenotipos de la enfermedad.

Métodos: estudiamos la frecuencia de las tres mutaciones del gen CARD15 (R702W, G908R, L1007fs) usando cebadores específicos, en un total de 216 pacientes con EC y 86 controles procedentes del área de Oviedo. Los pacientes fueron clasificados de acuerdo con la edad al diagnóstico, localización la enfermedad y su comportamiento clínico (clasificación de Viena).

Resultados: la frecuencia global de portadores de las mutaciones del gen CARD15 en los pacientes con EC fue del 17,3\% frente a un $17,6 \%$ en controles (NS). Al analizar separadamente los polimorfismos R702, G908R y L1007fs los pacientes mostraban frecuencias del 8,8, 3 y 6\% respectivamente, mientras que los controles las presentaban en el 11,6, 2,3 y 3,5\%, sin encontrar diferencias significativas para ninguna de ellas (NS). Las frecuencias observadas en controles, fueron similares a las encontradas en otras regiones españolas.

Conclusiones: la prevalencia de mutaciones en CARD15 en pacientes con EC en Asturias es menor a la reportada en otros trabajos publicados en población española. Otros factores ambientales, además de los genéticos, parecen tener mayor importancia en el desarrollo de EC en nuestra área.

Palabras clave: Enfermedad de Crohn (EC). Mutaciones del gen CARD15. Frecuencia de portadores. Fenotipos.

\begin{abstract}
Background: the association between the three common CARD15 gene mutations (R702W, G908R, L1007fs) and the genetic susceptibility to Crohn's disease (CD) have been confirmed by several studies, with some differences found, in relation to geographic areas and ethnic groups.

Objectives: To analyze the prevalence of CARD15 gen and its polymorphisms in patients with CD in Asturias and its possible correlation with the different genotypes of the disease.

Methods: a total of $216 \mathrm{CD}$ patients recruited from Asturias (North of Spain) and 86 ethnically matched healthy controls, were typed using Hybprobes on a LightCycler instrument for CARD15 mutations. Patients were subdivided according to Vienna classification. We have studied the frequency of these mutations in the different subgroups of $\mathrm{CD}$ patients and analyzed its contribution to the disease clinical characteristics and progression.

Results: carrier frequencies for CARD15 mutations in our CD patients were similar to controls (17.8 vs. $17.4 \%)$ respectively (NS). CD patients exhibited frequencies of $8.8,3.0$ and $6.0 \%$ for the R702, G908R and L1007fs polymorphisms respectively, whereas our control population had allele frequencies of 11.6, 2.3 and $3.5 \%$ for the three mutations respectively (NS).

We did not find any relationship between CARD15 mutations and the different phenotypes of Crohn's disease, according to $\mathrm{Vi}$ enna classification.

Conclusions: in our $\mathrm{CD}$ population, other factors (i.e. environmental), in addition to genetics, must be mainly involved in the development of the disease.
\end{abstract}

Key words: Crohn's disease. CARD15 mutations. Carrier frequencies. Fenotypes.

Rodrigo L, Martínez-Borra J, Garrote JA, Niño P, León AJ, Riestra S, Bernardo D, Barreiro M, Arranz E. Las mutaciones del gen CARD15 presentan escasa relación con los fenotipos de la enfermedad de Crohn en Asturias. Rev Esp Enferm Dig 2007; 99 : 570-575.

Recibido: 29-04-07.

Aceptado: 17-07-07.

Correspondencia: Luis Rodrigo. Servicio de Aparato Digestivo. Hospital Universitario Central de Asturias. C/ Celestino Villamil, s/n. 33006 Oviedo.e-mail: 1rodrigos@terra.es

\section{INTRODUCCIÓN}

La enfermedad de Crohn (EC) es un proceso inflamatorio gastrointestinal crónico complejo y clínicamente heterogéneo, en cuya patogenia están implicados tanto 
factores genéticos como ambientales. Se caracteriza por una respuesta inflamatoria inapropiada que conduce a la destrucción del tejido intestinal $(1,2)$. El gen de susceptibilidad para la EC más conocido es el CARD15 (CaspaseRecruitment-Domain-15), localizado en el cromosoma $16 \mathrm{q} 2(3,4)$. Se han descrito tres polimorfismos de un nucleótido localizado en la región $\mathrm{C}$ terminal del dominio LRR del gen CARD15: R702W (SNP8), G908R (SNP12) y L1007fs (SNP13) (3). Además otros genes (HLA) o interacciones con otras mutaciones, diferentes para cada grupo étnico, pueden influir en el fenotipo más que en la susceptibilidad (5-7).

La contribución de estas mutaciones a la susceptibilidad a la EC puede variar en diferentes áreas geográficas o grupos étnicos. Estos polimorfismos se han encontrado hasta en un 50\% de los pacientes con EC en Europa, y en un $20 \%$ de los individuos sanos (8) y su influencia en la susceptibilidad a la EC parece mayor en los países del Sur de Europa en comparación con los del Norte (8-17). Varios estudios clínicos realizados a nivel mundial, han puesto de manifiesto la asociación entre estas mutaciones del gen CARD15, con un incremento en la susceptibilidad a la EC, pero no con la colitis ulcerosa, estimándose la existencia de un riesgo medio relativo 2-3 veces mayor en los pacientes que tienen una variante alélica y entre 20-40 veces mayor, en los que presentan dos mutaciones simultáneas $(8,9,13,14,18-20)$.

Los objetivos del presente trabajo fueron determinar las frecuencias de las tres mutaciones más frecuentes de CARD15 en la población asturiana, y comprobar su valor diagnóstico y su significado clínico, como marcadores de EC.

\section{MATERIAL Y MÉTODOS}

\section{Pacientes y controles}

Hemos realizado un estudio de casos y controles, incluyendo un total de 216 pacientes con EC no relacionados, diagnosticados y tratados en los últimos veinte años en el Servicio de Digestivo del Hospital Universitario Central de Asturias en Oviedo; de ellos 114 eran varones y 102 mujeres, con una edad media $41 \pm 13$ años, que fueron seguidos durante un periodo medio de $12 \pm 4$ años. El diagnóstico de EC se realizó en base a diversos criterios clínicos, radiológicos, endoscópicos e histológicos y los pacientes fueron agrupados de acuerdo con los criterios de la clasificación de Viena (21). Así se subdividieron en las categorías de A1 o A2 (según que la aparición de la enfermedad fuese antes o después de los 40 años de edad, respectivamente); L1, L2, L3 y L4 (por la localización ileal, ileo-cólica, colónica y de tramos altos); por su comportamiento en B1, B2 o B3 (formas inflamatorias puras, estenosantes o fistulizantes). Las características clínicas de los pacientes con EC incluidos en el estudio, de acuerdo con los criterios de Viena, se muestran a continuación (Tabla I).

\begin{tabular}{llc}
\multicolumn{2}{c}{ Tabla I. Distribución de los pacientes con EC según } \\
clasificación de Viena \\
\hline Características clínicas & & $n(\%)$ \\
\hline Edad al diagnóstico & A1 (<40 años) & $188(87)$ \\
& A2 (> 40 años) & $28(13)$ \\
Localización & L1 (Ileal) & $67(31)$ \\
& L2 (Colónica) & $30(14)$ \\
& L3 (Íleo-cólica) & $114(53)$ \\
Forma & L4 (Intestino alto) & $5(2)$ \\
& B1 (Inflamatoria) & $82(38)$ \\
& B2 (Estenosante) & $37(17)$ \\
& B3 (Fistulizante) & $97(45)$ \\
\hline
\end{tabular}

Como controles se analizaron 86 muestras de donantes no relacionados, procedentes del Banco de Sangre de Oviedo. Se trataba de 46 mujeres (53\%) y 40 varones (47\%), con una edad media de $38 \pm 17$ años, en los que se excluyeron aquellos que refirieron antecedentes familiares de enfermedad inflamatoria intestinal.

Se obtuvo un consentimiento informado por escrito a todos los individuos que participaron en el estudio. Los datos clínicos y antecedentes relevantes fueron recogidos de forma protocolizada, $\mathrm{y}$ tratados de forma anónima mediante doble codificación: datos demográficos, clínica, histología y tratamientos (cirugía, esteroides, etc.). El estudio fue aprobado por el Comité Etico del Hospital Universitario Central de Asturias (HUCA).

\section{Genotipado}

Se obtuvieron muestras de sangre periférica anticoagulada con EDTA obtenidas por punción venosa. El DNA genómico fue aislado utilizando un kit comercial Ultra Clean DNA (MoBio Laboratorios, Solana Beach, California, EE.UU.), siguendo las instrucciones del fabricante. Se tiparon 3 polimorfismos del gen CARD15 (R702W, G908R, y L1007fs), utilizando cebadores Hyprobes en un Termociclador para q-PCR LightCycler (Roche, Manheim, Alemania), por análisis de las curvas de fusión de los híbridos formados entre los productos de la PCR (reacción en cadena de la polimerasa) y los oligonucleóticos específicos marcados con fluorocromos. Los cebadores y las sondas fluorescentes se diseñaron con el programa LightCycler ProbeDesign 2.0 (Tabla II) y fueron sintetizados por MWG Biotech (Ebersberg, Alemania). La técnica se estandarizó utilizando un panel de al menos tres muestras control diferentes para cada polimorfismo perfectamente caracterizadas, correspondientes a homocigotos de cada alelo y un heterocigoto. Las muestras control fueron suministradas por el Dr. Bart Crusius del Laboratorio de Inmunogenética Gastrointestinal de la Universidad Libre de Amsterdam (VUmc). El método tipó correctamente los 
Tabla II. Secuencias de oligonucleóticos utilizados para el tipaje de las tres mutaciones del gen CARD15 (las secuencias de oligonucleóticos están escritas en sentido de 5' a 3')

\begin{tabular}{lcl}
\hline Mutación & rs & Secuencias \\
\hline L1007fs & 2066847 & Hacia adelante: TCTTCTTTCCAGGTTGTCCAA \\
& & Hacia atrás: TGAGGTTCGGAGAGCTAAAACAG \\
& Anclaje: Cy5.5-CCATCCTGGAAGTCTGGTAAGGCC-Pho \\
& & Sensor: AGGCCCCTGAAAGGATGAC-Fluoresceína \\
G908R & \multirow{2}{*}{2066845} & Hacia adelante: ACACATATCAGGTACTCACTGACACT \\
& Hacia atrás: TTACCTGAGCCACCTCAAGC \\
& Anclaje: Cy5.5-TGAAAAGGCCAAAGAGTCAACAGAC-Pho \\
& Sensor: CCACTCTGTTGCGCCAGAA-Fluoresceína \\
R702W & Hacia adelante : AGCCGCACAACCTCAGATCAC \\
& Hacia atrás : GCGGGCACAGGCCTGGC \\
& Anclaje : Cy5.5-GTCTGGCACTCAGCCAGCAGGCCCC-Pho \\
& Sensor: GCGCCAGAGCAGGGCCTCTCA-Fluoresceína \\
\hline
\end{tabular}

controles positivos y en caso negativo se comprobó por secuenciación. Las reacciones de PCR se realizaron con un volumen total de $20 \mu \mathrm{l}$, y con una mezcla de PCR conteniendo de 50 a $100 \mathrm{ng}$ de DNA genómico, 0,5 $\mu \mathrm{M}$ de cada cebador, $0,1 \mu \mathrm{M}$ de cada sonda, $3 \mathrm{mM}$ de $\mathrm{MgCl}_{2}$, y $2 \mu l$ de DNA-Master Hibridación (Roche). Las condiciones de realización de las PCR para $2722 \mathrm{G}>\mathrm{C}$ y $2104 \mathrm{C}>\mathrm{T}$ fueron las siguientes: desnaturalización inicial a $95^{\circ} \mathrm{C}$ durante 2 minutos, seguido de 40 ciclos de desnaturalización a $95{ }^{\circ} \mathrm{C}$ por 1 segundo, anillado a 62 ${ }^{\circ} \mathrm{C}$ por 10 segundos y extensión a $72{ }^{\circ} \mathrm{C}$ durante 5 segundos. Tras la amplificación el análisis de las curvas de fusión se realizó por desnaturalización a $95{ }^{\circ} \mathrm{C}$ por $5 \mathrm{~s}$, naturalización a $50{ }^{\circ} \mathrm{C}$ por $10 \mathrm{~s}$, y un incremento de la temperatura hasta los $90{ }^{\circ} \mathrm{C}$ a una tasa de $0,1^{\circ} \mathrm{C} / \mathrm{s}$. Las condiciones de PCR para 3020 ins $\mathrm{C}$ fueron las mismas que las anteriores, salvo una temperatura de naturalización a $60{ }^{\circ} \mathrm{C}$. Siete pacientes con EC $(3,5 \%)$ fueron heterocigotos compuestos para las diversas mutaciones y no se encontraron entre los controles.

\section{Análisis estadístico}

Se construyeron tablas de contingencia, y se calculó el valor de la Chi cuadrado de Pearson, y la significación asintótica (p) y en las tablas 2 x 2 se aplicó la $\mathrm{Chi}^{2}$ con la corrección del test exacto, F de Fisher. Los riesgos fueron expresados mediante la Odds Ratio (OR) y el intervalo de confianza IC del 95\%. El análisis estadístico se realizó usando el programa SPSS v. 11.0. Las diferencias entre grupos fueron consideradas significativas cuando el valor de $\mathrm{p}$ fue menor de 0,05 .

\section{RESULTADOS}

Las frecuencias de portadores para las mutaciones de CARD15 estudiadas en pacientes con EC fueron similares a la de los controles: $17,3 \%(35 / 202)$ frente $17,6 \%$ $(15 / 85)$ respectivamente $\left(\mathrm{Chi}^{2}=0,001, \mathrm{p}=1, \mathrm{NS}\right)$. No encontramos diferencias estadísticamente significativas entre las frecuencias de genotipos, alelos o portadores entre nuestros pacientes con EC y los controles para ninguna de las mutaciones de CARD15 estudiadas (Tabla III).

Se encontró un caso homocigoto para la mutación R702W entre los pacientes con EC; sin embargo, no se encontró ningún homocigoto compuesto.

En relación con la edad de comienzo, en los pacientes jóvenes las mutaciones R702W y L1007FS fueron algo más frecuentes que en formas de comienzo más tardío, sin presentar diferencias significativas, mientras que la mutación G908R fue la más rara, apareciendo únicamente en tres pacientes de $155(1,9 \%)$ con diagnóstico anterior a los 40 años. Ningún paciente menor de 17 años presentó esta mutación.

El polimorfismo R702W fue el predominante en las formas con afectación de colon en un 9,3 frente a 5,6\% en las ileales. Por el contrario, las variantes G908R y L1007FS fueron las predominantes en las formas con

Tabla III. Frecuencia de portadores de las tres mutaciones del gen CARD15 en pacientes con EC en relación con el fenotipo de la enfermedad

\begin{tabular}{|c|c|c|c|c|c|c|c|c|c|c|}
\hline & \multicolumn{2}{|c|}{ CARD15 } & \multicolumn{2}{|c|}{$R 702 \mathrm{~W}$} & \multicolumn{2}{|c|}{ G908R } & \multicolumn{2}{|c|}{ L1007FS } & \multicolumn{2}{|c|}{ G908R or L1007FS } \\
\hline & $n$ & $\%$ & $n$ & $\%$ & $n$ & $\%$ & $n$ & $\%$ & $n$ & $\%$ \\
\hline Controles & $15 / 85$ & 17,4 & $10 / 86$ & 11,6 & $2 / 86$ & 2,3 & $3 / 86$ & 3,5 & $5 / 86$ & 5,8 \\
\hline $\begin{array}{l}\text { EC } \\
\text { Edad al diagnóstico (EC) }\end{array}$ & $35 / 202$ & 17,8 & $18 / 205$ & 8,8 & $6 / 202$ & 3 & $13 / 216$ & 6 & $19 / 207$ & 9 \\
\hline A1 & $24 / 153$ & 15,7 & $13 / 155$ & 8,4 & $3 / 155$ & 1,9 & $10 / 157$ & 6,4 & $13 / 157$ & 8,3 \\
\hline $\begin{array}{l}\text { A2 } \\
\text { Localización de la EC }\end{array}$ & $5 / 31$ & 16,1 & $2 / 32$ & 6,3 & $2 / 30$ & 6,7 & $1 / 38$ & 2,6 & $3 / 32$ & 9,3 \\
\hline L3 & $13 / 101$ & 12,9 & 9/103 & 8,7 & $3 / 101$ & 3 & $3 / 109$ & 2,8 & $6 / 104$ & 5,8 \\
\hline$L 1+L 3$ & $25 / 151$ & 16,6 & $12 / 153$ & 7,8 & $6 / 151$ & 4 & $9 / 162$ & 5,6 & $15 / 156$ & 9,6 \\
\hline $\begin{array}{l}\mathrm{L} 2+\mathrm{L} 3 \\
\text { Conducta de la EC }\end{array}$ & 19/137 & 13,9 & $13 / 140$ & 9,3 & $3 / 138$ & 2,2 & $5 / 145$ & 3,4 & $8 / 140$ & 5,7 \\
\hline B1 & $12 / 61$ & 19,7 & $8 / 62$ & 12,9 & $1 / 63$ & 1,6 & $4 / 64$ & 6,3 & $5 / 63$ & 7,9 \\
\hline B2 & $1 / 22$ & 4,5 & $0 / 25$ & 0 & $1 / 23$ & 4,3 & $0 / 25$ & 0 & $1 / 23$ & 4,3 \\
\hline
\end{tabular}


afectación ileal: el 17,3\% (9/52) de los pacientes L1 frente al 6\% (3/50) de portadores de R702W, y el 9,6\% (15/156) vs. el 7,8\% (12/153) respectivamente en L1 + L3.

La enfermedad perianal activa (EPA) no se asoció con la presencia de alguna de las mutaciones (8\% con EPA frente a $10,6 \%$ sin EPA). No encontramos ningún patrón especialmente relacionado con la edad de comienzo, localización, ni con el comportamiento clínico de la enfermedad.

\section{DISCUSIÓN}

No se encontraron diferencias en la frecuencia de mutaciones en CARD15 entre pacientes con EC y controles $(17,3$ vs. $17,6 \%)$. Por lo tanto, la prevalencia de mutaciones en el gen CARD15 es mucho menor en nuestros pacientes comparados con estudios previos llevados a cabo con pacientes de EC realizados en diferentes regiones españolas siendo del 32,8\% en Madrid (22) y del 27,9\% en Galicia (23); siendo algo más parecidas a las encontradas en Toledo del 15,2\% (24). Sin embargo, la frecuencia de portadores de al menos una mutación entre los controles sanos de la población asturiana, resultó similar a la de la población control gallega (17,6 y 15,2\%), y algo mayor que la descrita en Madrid (10,7\%). La distribución de frecuencias para cada mutación fue también similar entre las poblaciones control asturiana y gallega, como era de esperar por la proximidad geográfica, aunque con un ligero aumento en la prevalencia de las mutaciones G908R y L1007FS.

La asociación entre las mutaciones de CARD15 y la aparición o desarrollo de la EC se ha confirmado en diferentes áreas geográficas y grupos étnicos $(5,25)$. En las poblaciones caucásicas europeas y norteamericanas, menos del $50 \%$ de los pacientes con EC presentan la mutación CARD15 y entre el 10 y el 20\% de los individuos sanos son portadores de estas mutaciones. Por lo tanto, la presencia de las mutaciones en el mencionado gen no se consideran, ni necesarias ni suficientes, como factores condicionantes para el desarrollo y aparición de una EC, explicando sólo el $20 \%$ de la susceptibilidad genética a la EC. En Europa existe un gradiente de la contribución de las mutaciones de CARD15 a esta susceptibilidad. Así en los países del Norte de Europa su prevalencia es menor $(10,11,15-17)$ que en el resto de los países europeos $(8,9,12-14)$. Sin embargo en Asia, África Subsahariana y Sudamérica, estas mutaciones son raras o están ausentes $(18,19,26-28)$, y en todas estas áreas la enfermedad es mucho menos prevalente que en Europa o Estados Unidos.

En general, las diferencias encontradas en distintos estudios pudieran deberse a variaciones étnicas entre poblaciones, cambios en las definiciones fenotípicas y a un reducido número de casos por grupo estudiado (29). Algunas de las diferencias en las frecuencias de portadores entre los pacientes con EC observadas en algunas áreas de Europa no pueden ser explicadas sólo por diferencias genéticas, sino más bien por factores ambientales, dada la similitud de las frecuencias entre los grupos de población sana. Es más, la existencia de estas diferencias entre poblaciones obliga a la realización de los estudios genéticos en las distintas áreas geográficas para determinar el papel relativo de estas mutaciones en la susceptibilidad a la EC en cada área.

La población española es bastante heterogénea. Las diferentes regiones tienen distintos orígenes y en su devenir histórico tuvieron varias y diversas invasiones de poblaciones extranjeras que se asentaron y mezclaron con su población. Sin embargo Asturias, debido a su localización, limitada por el mar Cantábrico al norte y por los Picos de Europa al sur, permaneció relativamente aislada. Esta podría ser la causa de la ausencia de diferencias entre casos y controles en relación con las mutaciones del CARD15, comportándose de manera similar a otras poblaciones aisladas en Asia $(18,26)$. Por otra parte, diferencias dentro del mismo país se han encontrado también en Gran Bretaña, en estudios realizados en poblaciones relativamente heterogéneas $(9,14)$, en los que, de forma general, la presencia de mutaciones fue similar a la encontrada en otras poblaciones caucásicas, pero en poblaciones más homogéneas como los escoceses o los irlandeses, la presencia de mutaciones en CARD15 en la EC fue mucho más limitada (30).

La incidencia de EC en España está comprendida entre 0,4 y 5,5 casos/100.000 hab./año (31), habiéndose sugerido que la tasa de incidencia global ajustada en España estaría situada en una posición intermedia entre la de los países del Norte y del Sur de Europa (24). Los estudios genéticos realizados en Madrid (22) y Galicia (23) muestran una frecuencia de portadores de mutaciones del CARD15 de 32,8 y $27 \%$ en pacientes con EC, y de 10,7 y $15 \%$ respectivamente en controles. En Asturias la incidencia de EC es ligeramente mayor que la media nacional, siendo de 7,5 casos por 100.000 hab./año (IC95\%: 3,8-11,2) de tasa global, similar a la observada en países del Norte de Europa (32).

La mutación L1007FS resultó ser la observada con mayor frecuencia tanto entre pacientes como en controles en Asturias, aunque sin alcanzar diferencias estadísticamente significativas ( 6 frente a $3,5 \%, \mathrm{Chi}^{2}=0,785, \mathrm{p}=0,375$ ). Sin embargo, la frecuencia de portadores de otras mutaciones como la R702W (8,8 vs. 11,5\%) y la G908R (3vs. $2,3 \%$ ) fue muy similar entre pacientes y controles. En pacientes de otras zonas de España, las frecuencias de portadores para cada mutación individual fueron algo más elevadas, entre 13,3 y $13,7 \%$ para $\mathrm{R} 702 \mathrm{~W}$, entre 7,9 y $8,3 \%$ para G908R y entre 8,5 y $14,2 \%$ para L107FS, mientras que en controles, el rango de frecuencias osciló entre 4,3 y $11,5 \%$, entre 2,1 y $1,8 \%$ y entre 4,3 y $1,8 \%$ para R702W, G908R y L1007FS, respectivamente. En uno de los estudios (22) se encontró una relación positiva entre la presencia de mutaciones de CARD15 y la localización ileal de la enfermedad, en concordancia con otros estudios multicéntricos, que incluyen poblaciones más heterogéneas y no observaron esta asociación. 
No se encontraron diferencias significativas entre las distribuciones de frecuencias de portadores con respecto a la edad de aparición de la enfermedad, pero se encontró por lo general una mayor incidencia de portadores R702W y L1007FS entre los pacientes más jóvenes. Se discute la relación entre la presencia de mutaciones de CARD15 y presentación temprana de la enfermedad. Es posible que el límite de los 40 años de la clasificación de Viena resulte inadecuado para medir este efecto. Otro problema es que la edad al diagnóstico sólo se recoge en algunos de los estudios publicados. A pesar de todo, varios estudios publicados sí que encuentran relación entre dos de las mutaciones y el inicio temprano de la EC $(8,9,11)$. Asímismo, pacientes portadores de mutaciones del CARD15 presentan más frecuentemente enfermedad con localización ileal o íleo-cólica (L1, L3) $(9,13,15,33$ 36), pero no con la presentación exclusivamente de colon (ni con la CU o colitis indeterminada) $(13,35)$.

Los portadores de las mutaciones G908R o L1007FS fueron predominantes en las presentaciones de tipo estenosante (B2) o penetrante (B3), o con localización exclusivamente ileal (L1), mientras que la mutación R702W fue más frecuente en presentaciones afectando al colon. Por otra parte, los pacientes con formas inflamatorias (no estenosantes-no penetrantes, B1) portaban más frecuentemente la mutación R702W. El fenotipo más común en el momento del diagnóstico es el inflamatorio, y varios factores pueden determinar el desarrollo de complicaciones como fístulas y fibroestenosis $(36,37)$. La localización de la enfermedad puede ser un factor determinante para la evolución a este tipo de complicaciones $(28,38)$. Los pacientes con mutaciones en CARD15, particularmente con la SNP13 (L1007fs), tienen más probabilidad de desarrollar un fenotipo caracterizado por enfermedad localizada a nivel del íleon terminal, comienzo juvenil y presentar complicaciones de tipo fibroestenótico $(36,39)$. Es más, en niños con CD, la necesidad de cirugía y las complicaciones quirúrgicas precoces $(17,40)$ se asocian con la presencia de L1007fs, y esta mutación puede usarse como marcador predictivo de necesidad de cirugía (41-43). Estos factores genéticos pueden ser relevantes para la progresión de la enfermedad (un proceso cambiante en función del tiempo). Por tanto, la presencia de mutaciones de CARD15 podría no reflejar el fenotipo de la enfermedad en el momento del diagnóstico, sino junto con otros factores, podrían influir en la progresión de la enfermedad $(5,10)$.

El gen CARD15 codifica la proteína citoplasmática NOD-2 (Nucleotide Binding Oligomerization Domain-2), principalmente expresada en células de Paneth y fagocitos mononucleares, con una función biológica aún no totalmente aclarada. La expresión predominante de NOD-2 por las células de Paneth, podría explicar la asociación de los polimorfismos de CARD15 y la enfermedad ileal, donde estas células son muy abundantes $(44,45)$. El ligando del NOD-2, el MDP (Muramil Di-Péptido), se genera tras la digestión lisosómica del peptidoglicano, el componente principal de la pared bacteriana. El NOD-2 y los TLR (toll-like receptors), ambos miembros de la familia de "receptores de reconocimiento de patrones" (pattern-recognition receptors, o PRR), parecen actuar de forma sinérgica a nivel de las vías de activación de diversas citoquinas proinflamatorias $(46,47)$. Las células portadoras de las formas mutantes del NOD-2 podrían tener una capacidad alterada de inducción de la activación del NF-к $\beta$ (Factor Nuclear Kappa Beta) ante la estimulación con MDP (48).

Aunque las mutaciones del CARD15 son factores de riesgo para EC conocidos en la población caucásica, su uso como prueba diagnóstica no proporciona una información clínica relevante para la toma de decisiones. Las pruebas genéticas deben realizarse cuando aportan beneficios claros para el paciente: mejor caracterización, selección de tratamiento adecuado según la localización o las complicaciones, etc. Con estas premisas, la detección de mutaciones de CARD15 tienen escaso valor como pruebas de apoyo diagnóstico y muy dudoso como marcador de pronóstico en nuestra población asturiana. Quizás, otros genes de susceptibilidad o factores ambientales podrían estar interviniendo en el desarrollo de la EC en nuestra área geográfica.

\section{AGRADECIMIENTOS}

Este trabajo ha sido parcialmente financiado con ayudas del Instituto de Salud Carlos III, Ministerio de Sanidad (02/3068), Becas del Ministerio de Educación (FPU, AP2002-2696), Junta de Castilla y León (O.C.14/11/03O.R.26/01/0), y Phadia (Sweden Diagnostics afiliada a Pharmacia Diagnostics).

\section{BIBLIOGRAFÍA}

1. Baumgart DC, Carding SR. Inflammatory bowel disease: cause and immunobiology. Lancet 2007; 369: 1627-40.

2. Bouma G, Strober W. The immunological and genetic basis of inflammatory bowel disease. Nat Rev Immunol 2003; 3: 521-33.

3. Shanahan F. Crohn's disease. Lancet 2002; 359: 62-9.

4. Hugot JP, Chamaillard M, Zouali H, Lesage S, Cezard JP, Belaiche J, et al. Association of NOD2 leucine-rich repeat variants with susceptibility to Crohn's disease. Nature 2001; 411: 599-603.

5. Ogura Y, Bonen DK, Inohara N, Nicolae DL, Chen FF, Ramos R, et al. A frameshift mutation in NOD2 associated with susceptibility to Crohn's disease. Nature 2001; 411: 603-6.

6. Gaya DR, Russell RK, Nimmo ER, Satsangi J. New genes in inflammatory bowel disease: Lessons for complex diseases? Lancet 2006; 367: $1271-84$

7. Silverberg MS, Mirea L, Bull SB, Murphy JE, Steinhart AH, Greenberg GR, et al. A population- and family-based study of Canadian families reveals association of HLA DRB $1 * 0103$ with colonic involvement in inflammatory bowel disease. Inflamm Bowel Dis 2003; 9: $1-9$.

8. Yap LM, Ahmad T, Jewell DP. The contribution of HLA genes to IBD susceptibility and phenotype. Best Pract Res Clin Gastroenterol 2004: 18 : 577-96.

9. Lesage S, Zouali H, Cezard JP, Colombel JF, Belaiche J, Almer S, et al. CARD15/NOD2 mutational analysis and genotype-phenotype correlation in 612 patients with inflammatory bowel disease. Am J Hum Genet 2002; 70: 845-57. 
10. Ahmad T, Armuzzi A, Bunce M, Mulcahy-Hawes K, Marshall SE, Orchard TR, et al. The molecular classification of the clinical manifestations of Crohn's disease. Gastroenterol 2002; 122: 854-66.

11. Arnott ID, Satsangi J. Crohn's disease or Crohn's diseases? Gut 2003; 52: 460-1.

12. Bairead E, Harmon DL, Curtis AM, Kelly Y, O'Leary C, Gardner M, et al. Association of NOD2 with Crohn's disease in a homogenous Irish population. Eur J Hum Genet 2003; 11: 237-44.

13. Buning C, Genschel J, Buhner S, Kruger S, Kling K, Dignass A, et al. Mutations in the NOD2/CARD15 gene in Crohn's disease are associated with ileocecal resection and are a risk factor for reoperation. Aliment Pharmacol Ther 2004; 19: 1073-8.

14. Cuthbert AP, Fisher SA, Mirza MM, King K, Hampe J, Croucher PJ, et al. The contribution of NOD2 gene mutations to the risk and site of disease in inflammatory bowel disease. Gastroenterol 2002; 122: 867-74.

15. Hampe J, Cuthbert A, Croucher PJ, Mirza MM, Mascheretti S, Fisher S, et al. Association between insertion mutation in NOD2 gene and Crohn's disease in German and British populations. Lancet 2001; 357: 1925-8

16. Helio T, Halme L, Lappalainen M, Fodstad H, Paavola-Sakki P, Turunen $\mathrm{U}$, et al. CARD15/NOD2 gene variants are associated with familially occurring and complicated forms of Crohn's disease. Gut 2003; 52: 558-62.

17. Idestrom M, Rubio C, Granath F, Finkel Y, Hugot JP. CARD15 mutations are rare in Swedish pediatric Crohn disease. J Pediatr Gastroenterol Nutr 2005; 40: 456-60.

18. Russell RK, Drummond HE, Nimmo EE, Anderson N, Smith L, Wilson DC, et al. Genotype-phenotype analysis in childhood-onset Crohn's disease: NOD2/CARD15 variants consistently predict phenotypic characteristics of severe disease. Inflamm Bowel Dis 2005; 11: $955-64$.

19. Bonen DK, Ogura Y, Nicolae DL, Inohara N, Saab L, Tanabe T, et al. Crohn's disease-associated NOD2 variants share a signaling defect in response to lipopolysaccharide and peptidoglycan. Gastroenterol 2003; 124: 140-6.

20. Inoue N, Tamura K, Kinouchi Y, Fukuda Y, Takahashi S, Ogura Y, et al. Lack of common NOD2 variants in Japanese patients with Crohn's disease. Gastroenterol 2002; 123: 86-91.

21. Vermiere S, Louis E, Rutgeerts P, De Vos M, Van Gossum A, Belaiche $\mathrm{J}$, et al. NOD2/CARD15 does not influence response to infliximab in Crohn's disease. Gastroenterol 2002; 123: 106-11.

22. Gasche C, Scholmerich J, Brynskov J, D'Haens G, Hanauer SB, Irvine EJ, et al. A simple classification of Crohn's disease: Report of the Working Party for the World Congresses of Gastroenterology, Vienna 1998. Inflamm Bowel Dis 2000; 6: 8-15.

23. Mendoza JL, Murillo LS, Fernandez L, Pena AS, Lana R, Urcelay E, et al. Prevalence of mutations of the NOD2/CARD15 gene and relation to phenotype in Spanish patients with Crohn's disease. Scand J Gastroenterol 2003; 38: 1235-40

24. Núñez C, Barreiro M, Dominguez-Muñoz JE, Lorenzo A, Zapata C, Peña AS. CARD15 mutations in patients with Crohn's disease in a homogeneous Spanish population. Am J Gastroenterol 2004; 99: 450-6.

25. De Diego C, Alcántara M, Valle J, Perez-Grueso MJ, Munoz-Rosas C, Carrobles JM, et al. Frequency of CARD15 polymorphisms in patients with Crohn's disease from Toledo, Spain: genotype-phenotype correlation. Genet Test 2006; 10: 178-85.

26. Silverberg MS, Satsangi J, Ahmad T, Arnott ID, Bernstein CN, Brant $\mathrm{SR}$, et al. Toward an integrated clinical, molecular and serological classification of inflammatory bowel disease: Report of a Working Party of the 2005 Montreal World Congress of Gastroenterology. Can J Gastroenterol 2005; 19 (Supl. A): 5-36.

27. Croucher PJ, Mascheretti S, Hampe J, Huse K, Frenzel H, Stoll M, et al. Haplotype structure and association to Crohn's disease of CARD15 mutations in two ethnically divergent populations. Eur J Hum Genet 2003; 11: 6-16.

28. Leong RW, Armuzzi A, Ahmad T, Wong ML, Tse P, Jewell DP, et al. NOD2/CARD15 gene polymorphisms and Crohn's disease in the Chinese population. Aliment Pharmacol Ther 2003; 17: 1465-70.
29. Yamazaki K, Takazoe M, Tanaka T, Kazumori T, Nakamura Y. Absence of mutation in the NOD2/CARD15 gene among 483 Japanese patients with Crohn's disease. J Hum Genet 2002; 47: 469-72.

30. Cosnes J, Cattan S, Blain A, Beaugerie L, Carbonnel F, Parc R, et al. Long-term evolution of disease behavior of Crohn's disease. Inflamm Bowel Dis 2002; 8: 244-50.

31. Arnott ID, Nimmo ER, Drummond HE, Fennell J, Smith BR, MacKinlay E, et al. NOD2/CARD15, TLR4 and CD14 mutations in Scottish and Irish Crohn's disease patients: evidence for genetic heterogeneity within Europe? Genes Immun 2004; 5: 417-25.

32. Pajares JM, Gisbert JP. Epidemiology of inflammatory bowel disease in Spain. A systematic review. Rev Esp Enferm Dig 2001; 93: 9-20.

33. Rodrigo L, Riestra S, Niño P, Cadahia V, Tojo R, Fuentes D, et al. A population-based study on the incidence of inflammatory bowel disease in Oviedo (Northern Spain). Rev Esp Enferm Dig 2004; 96: 296305

34. Gasche C, Alizadeh BZ, Pena AS. Genotype-phenotype correlations: how many disorders constitute inflammatory bowel disease? Eur J Gastroenterol Hepatol 2003; 15: 599-606.

35. Hampe J, Grebe J, Nikolaus S, Solberg C, Croucher PJ, Mascheretti $\mathrm{S}$, et al. Association of NOD2 (CARD 15) genotype with clinica course of Crohn's disease: A cohort study. Lancet 2002; 359: 1661-5.

36. Hugot JP, Zouali H, Lesage S. Lessons to be learned from the NOD2 gene in Crohn's disease. Eur J Gastroenterol Hepatol 2003; 15: 593-7.

37. Vavassori P, Borgiani P, D'Apice MR, De Negris F, Del Vecchio Blanco G, Monteleone I, et al. 3020insC mutation within the NOD2 gene in Crohn's disease: frequency and association with clinical pattern in an Italian population. Dig Liver Dis 2002; 34: 153

38. Abreu MT, Taylor KD, Lin YC, Hang T, Gaiennie J, Landers CJ, et al. Mutations in NOD2 are associated with fibrostenosing disease in patients with Crohn's disease. Gastroenterology 2002; 123: 679-88.

39. Radlmayr M, Torok HP, Martin K, Folwaczny C. The c-insertion mutation of the NOD2 gene is associated with fistulizing and fibrostenotic phenotypes in Crohn's disease. Gastroenterology 2002; 122 : 2091-2.

40. Louis E, Michel V, Hugot JP, Reenaers C, Fontaine F, Delforge M, et al. Early development of stricturing or penetrating pattern in Crohn's disease is influenced by disease location, number of flares, and smoking but not by NOD2/CARD15 genotype. Gut 2003; 52: 552-7.

41. Economou M, Trikalinos TA, Loizou KT, Tsianos EV, Ioannidis JP Differential effects of NOD2 variants on Crohn's disease risk and phenotype in diverse populations: A metaanalysis. Am J Gastroenterol 2004; 99: 2393-404.

42. Kugathasan S, Collins N, Maresso K, Hoffmann RG, Stephens M, Werlin SL, et al. CARD15 gene mutations and risk for early surgery in pediatric-onset Crohn's disease. Clin Gastroenterol Hepatol 2004; 2: 1003-9.

43. Barreiro M, Núñez C, Dominguez-Muñoz JE, Lorenzo A, Barreiro F, Potel J, et al. Association of NOD2/CARD15 mutations with previous surgical procedures in Crohn's disease. Rev Esp Enferm Dig 2005; 97: 547-53.

44. Inohara N, Ogura Y, Fontalba A, Gutierrez O, Pons F, Crespo J, et al Host recognition of bacterial muramyl dipeptide mediated through NOD2. Implications for Crohn's disease. J Biol Chem 2003; 278 : 5509-12.

45. Lala S, Ogura Y, Osborne C, Hor SY, Bromfield A, Davies S, et al Crohn's disease and the NOD2 gene: a role for paneth cells. Gastroenterology 2003; 125: 47-57.

46. Ogura Y, Lala S, Xin W, Smith E, Dowds TA, Chen FF, et al. Expression of NOD2 in Paneth cells: A possible link to Crohn's ileitis. Gut 2003; 52: 1591-7.

47. Li J, Moran T, Swanson E, Julian C, Harris J, Bonen DK, et al. Regulation of IL-8 and IL-1beta expression in Crohn's disease associated NOD2/CARD15 mutations. Hum Mol Genet 2004; 13: 1715-25.

48. Van Heel DA, Ghosh S, Butler M, Hunt KA, Lundberg AM, Ahmad $\mathrm{T}$, et al. Muramyl dipeptide and toll-like receptor sensitivity in NOD2-associated Crohn's disease. Lancet 2005; 365: 1794-6. 